\section{Groove pancreatitis: A series of 5 cases}

Groove pancreatitis is a distinct form of chronic pancreatitis affecting the region between the duodenum, head of pancreas and the common bile duct. ${ }^{1}$ The exact incidence of groove pancreatitis is not known, but it accounts for approximately $19.5 \%-24.4 \%$ of pancreaticoduodenectomies performed to treat chronic pancreatitis. ${ }^{2}$ Patients usually present with pancreatic type of chronic abdominal pain. The major differential to this condition would be peri-ampullary carcinoma or carcinoma of the head of pancreas. The diagnosis is more easily made radiologically than clinically. We present a series of 5 cases of groove pancreatitis that presented to our tertiary referral hospital in the past 6 months.

\section{Case Reports}

This series consists of 5 patients who presented with abdominal pain and had either normal levels or an insignificant rise of pancreatic enzymes. Their clinical and other features are shown in Table 1. The diagnosis of pancreatitis was made on the basis of pancreatic type of pain and the CT findings. ${ }^{3}$ All patients were middleaged males (mean age: $46 \mathrm{yrs}$ ) presenting with recurrent abdominal pain, for durations varying from 3 months to 7 years. Loss of weight was significant in almost all the patients. One of the patients had earlier been treated for chronic pancreatitis with a Beger's duodenum-preserving pancreatic head resection that resulted in cessation of symptoms for 5 to 6 years with recurrence thereafter, for which celiac plexus block was performed which gave him symptomatic relief for 5 months. Another patient underwent celiac plexus blockwith splanchnicectomy for pain relief but was lost to follow up. Two of the patients were diabetic and three of them consumed alcohol in significant amounts.

\section{Discussion}

Groove pancreatitis is a relatively uncommon condition that is not very well recognized. This condition was first described by Becker et al in $1973 .{ }^{3}$ Due to the overlap of many differential diagnoses such as heterotopic pancreas, pancreatic hematoma, and periampullary maliganancies, Adsay and Zamboni proposed the term "paraduodenal pancreatitis" for this condition. ${ }^{4}$

The exact pathogenesis of groove pancreatitis remains unclear. The most widely accepted pathogenic mechanisms for groove pancreatitis is that it could arise due to an anatomical or functional obstruction of the minor papilla and/or an increased viscosity of the pancreatic juices caused by factors such as smoking and alcohol, which could lead to stasis of pancreatic juices causing pancreatitis. Other mechanisms include fibrous scarring due to peptic ulcer, duodenal infections or duodenal wall cyst. $^{2}$

To establish a definitive diagnosis of groove pancreatitis is a daunting task. Patients with groove pancreatitis usually present with chronic abdominal pain, nausea with vomiting and weight loss. Jaundice is not a prominent symptom unless there is a common bile duct stricture. Blood tests might show a slight elevation in the pancreatic and hepatic enzymes or tumor markers carbohydrate antigen 19-9 or carcinoembryonic antigen; however these elevation are present only in few and thus are seldom useful. Upper gastrointestinal endoscopy is normal or may show an inflamed mucosa or stenosis of the duodenal lumen. ${ }^{5}$

Various imaging modalities are present for the diagnosis of groove pancreatitis, which include transabdominal ultrasonography, magnetic resonance imaging (MRI), computed tomography (CT) and endoscopic ultrasound (EUS). On abdominal ultrasonography, a hypoechoic mass is often noted adjacent to the pancreatic head with thickening of the second part of the duodenum and widening of the periduodenal interface with the pancreatic head. On a CT scan, paraduodenal pancreatitis can be manifest in four forms1: 1) Solid variant - A hypoattenuating poorly enhancing soft tissue mass in the pancreatico-duodenal groove with or without calcific specks. 2) Cystic variant - cysts in the duodenal wall and/or the groove may occur in isolation or as part of the solid variant. 3) Pure form - the appearance can range from ill-defined fat stranding and inflammatory change in the groove between the pancreatic head and 
Table 1: Clinical and demographic features of the 5 patients

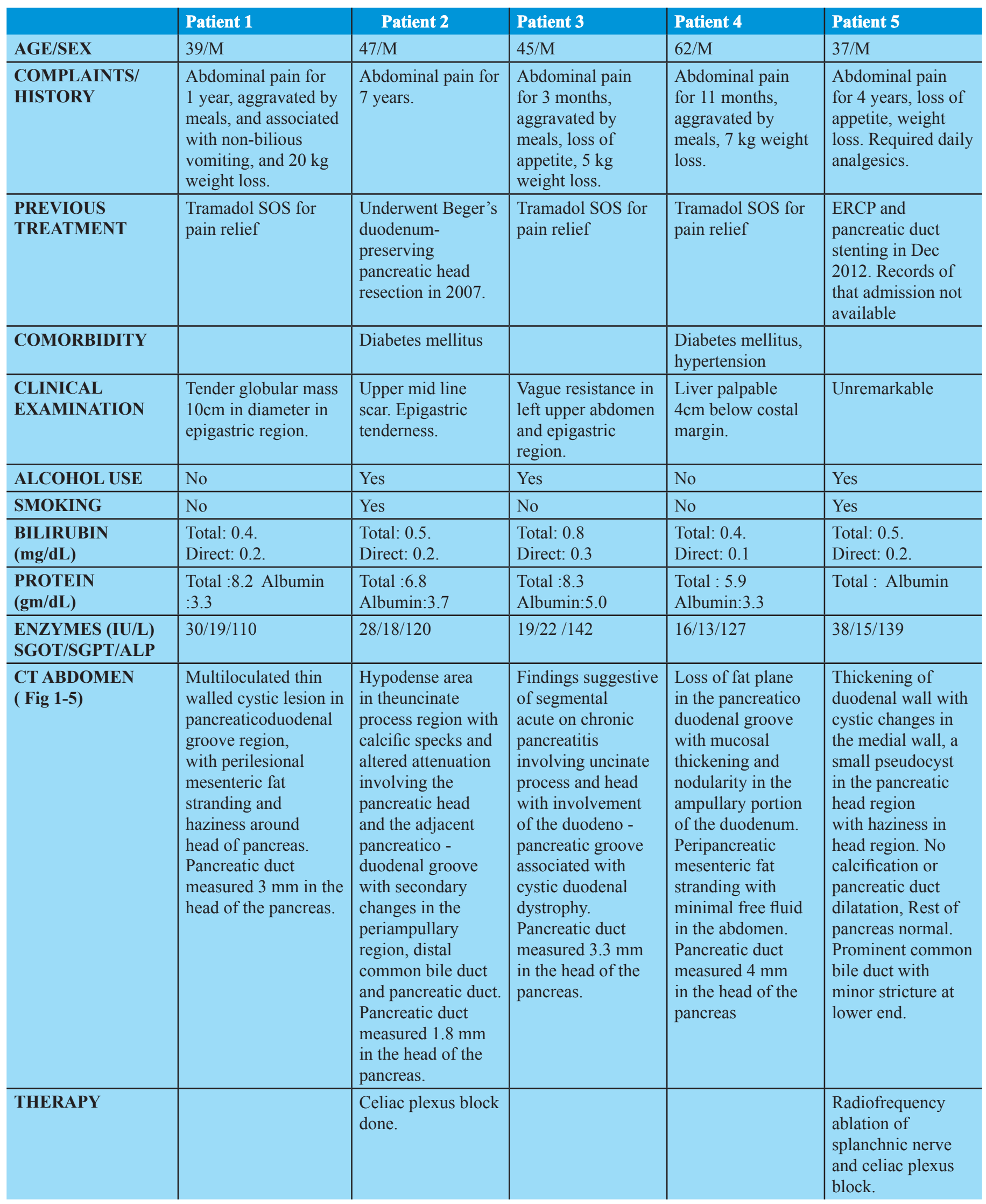




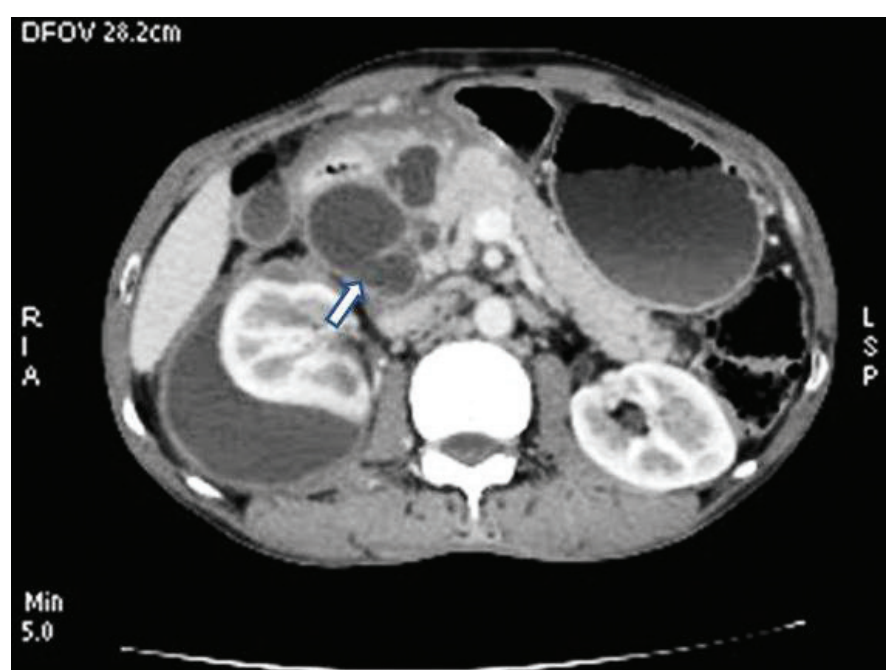

Figure 1: Axial multidetector contrast enhanced computed tomography (CECT) scan showing cysts with inflammatory changes.

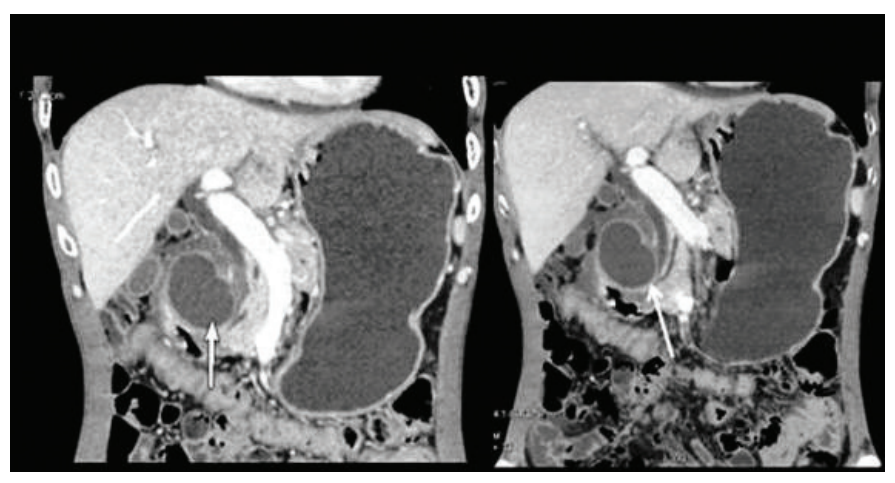

Figure 2: Axial and coronal multidetector CECT image showing inflammatory changes in the groove with cyst formation displacing the common channel (arrow) medially and the second part of the duodenum laterall.

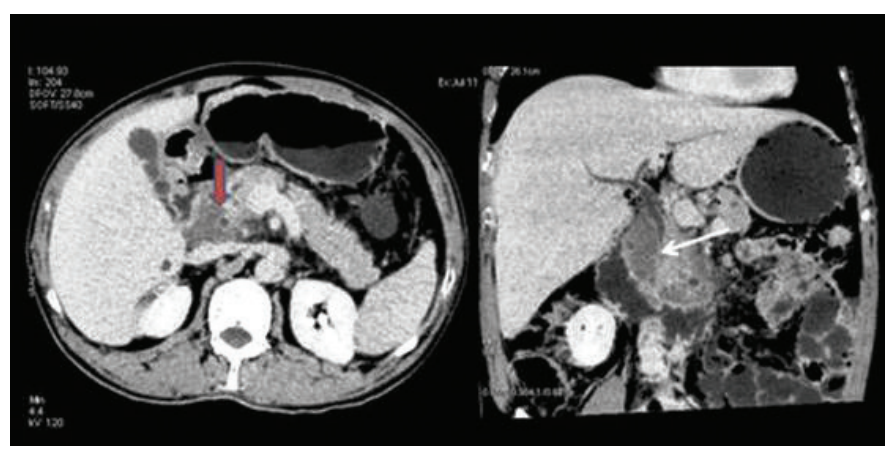

Figure 3: Axial CECT delayed images showing hypodense soft tissue in the groove (thick arrow) with smooth tapering of the distal common bile duct (thin arrow).

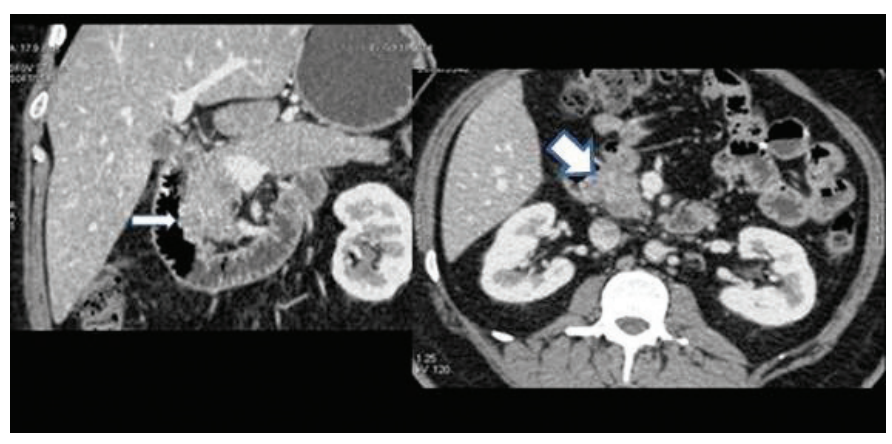

Figure 4: and axial CECT images showing a sheet of heterogeneously enhancing soft tissue in the groove with secondary changes in the second part of the duodenum (arrow).

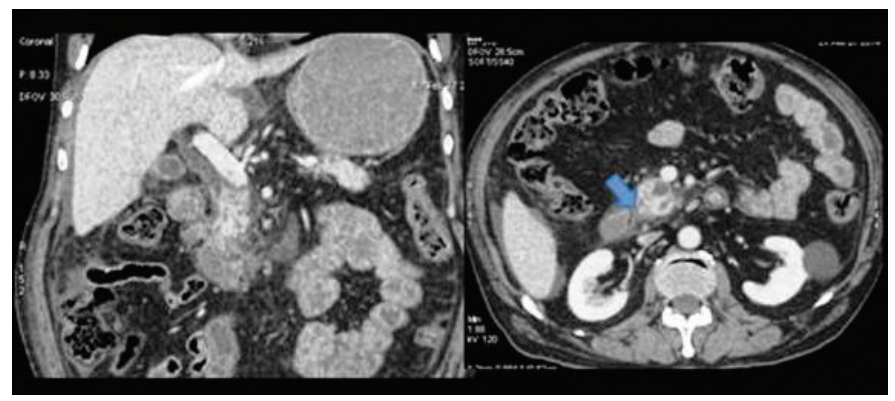

Figure 5: Coronal and axial CECT images showing irregular sheet of soft tissue in the groove (arrow).

duodenum, to frank crescentic soft tissue in the groove. 4) Segmental form - mass like enlargement of the pancreatic head is seen this form which is commonly confused with a pancreatic head mass. Ductal dilation is uncommon in groove pancreatitis, thus distinguishing it from pancreatic adenocarcinoma. MRI with MR cholangiopancreaticography (MRCP) shows a sheet-like mass corresponding to the fibrous scar in the groove, which is hypointense to the pancreas on T1W images with minimal delayed enhancement on contrast enhanced MR. Cysts appear as T2 hyperintense areas. Smooth long segment common bile duct tapering can be also noted. ${ }^{6}$

Treatment of groove pancreatitis depends on the clinical presentation. Patients who present acutely are managed similar to those of acute pancreatitis. There are reports of pain relief after minor duct stenting but such treatments are often temporary. Surgery is the preferred approach because it may give a prolonged pain relief 
and helps to rule out a pancreatic head malignancy. Surgical procedures comprise pancreatoduodenectomy (Whipple procedure) or pylorus-preserving pancreatoduodenectomy. ${ }^{7}$

Groove pancreatitis is often treated as chronic pancreatitis without recognition of its features, as seen in one of our patients. We present this series of five cases to draw attention to this relatively uncommon condition, which can be more easily diagnosed with advances in radiological imaging.

DORAISWAMI BABU VINISH D. KARTHIKEYAN ARULPRAKASH S DINESH RAMASWAMY SENTHIL GANESAN P. RADHAKRISHNA B.S. RAMAKRISHNA

Institute of Gastroenterology \& Department of Radiology, SRM Institutes for Medical Science, Chennai, Tamil Nadu, India.

Correspondence: Doraiswami Babu Vinish Email:vinishbabu@rediffmail.com

\section{References}

1. Raman SP, Salaria SN, Hruban RH etal. Groove pancreatitis: Spectrum of imaging findings and radiology-pathology correlation. Am J Roentgenol. 2013;201:W29-W39.

2. Tezuka K, Makino T, Hirai I et al. Groove pancreatitis. Dig Surg. 2010;27:149-152.

3. Becker V. Bauchspeicheldrüse. In: Doerr W, Seifert G, Uehlinger E (eds): Spezielle Pathologische Anatomie. Vol. 6. Berlin, Springer, 1973.

4. Adsay NV, Zamboni G. Paraduodenal pancreatitis: a clinico-pathologically distinct entity unifying 'cystic dystrophy of heterotopic pancreas', 'para-duodenal cyst', and 'groove pancreatitis'. Semin Diagn Pathol 2004; 21:247-254.

5. Shudo R, Yazaki Y, and Sakurai S et al. Groove pancreatitis: report of a case and review of the clinical and radiological features of groove pancreatitis reported in Japan. Intern Med. 2002;41:537-542.

6. Blasbalg R, Baroni RH, Costa DN et al. MRI features of groove pancreatitis. Am J Roentgenology. 2007;189:73-80.

7. Black TP, Guy CD, White RR et al. Groove pancreatitis: Four cases from a single center and brief review of the literature. ACG Case Rep J. 2014;1:154-157. 